\title{
Gamma Knife radiosurgery for meningiomas in patients with neurofibromatosis Type 2
}

\author{
Ann Liu, BS, ${ }^{1}$ Elizabeth N. Kuhn, MD, ${ }^{1}$ John T. Lucas Jr., MSCR, MD, ${ }^{2}$ Adrian W. Laxton, MD, ${ }^{1}$ \\ Stephen B. Tatter, MD, PhD, ${ }^{1}$ and Michael D. Chan, MD²
}

Departments of ${ }^{1}$ Neurosurgery and ${ }^{2}$ Radiation Oncology, Wake Forest School of Medicine, Winston-Salem, North Carolina

OBJECT Neurofibromatosis Type 2 (NF2) is a rare autosomal dominant disorder predisposing patients to meningiomatosis. The role of stereotactic radiosurgery (SRS) is poorly defined in NF2, and although the procedure has excellent control rates in the non-NF2 population, its utility has been questioned because radiation has been hypothesized to predispose patients to malignant transformation of benign tumors. To the authors' knowledge, this is the first study to examine the use of SRS specifically for meningiomas in patients with NF2.

METHODS The authors searched a tumor registry for all patients with NF2 who had undergone Gamma Knife radiosurgery (GKRS) for meningioma in the period from January 1, 1999, to September 19, 2013, at a single tertiary care cancer center. Medical records were retrospectively reviewed for patient and tumor characteristics and outcomes.

RESULTS Among the 12 patients who met the search criteria, 125 meningiomas were identified, $87(70 \%)$ of which were symptomatic or progressive and thus treated with GKRS. The median age at the first GKRS was 31 years (interquartile range [IQR] 27-37 years). Five patients (42\%) had multiple treatments with a median of 27 months (IQR 14-50 months) until the subsequent GKRS. The median follow-up in surviving patients was 43 months (IQR 34-110 months). The 5 -year local tumor control and distant treatment failure rates were $92 \%$ and $77 \%$, respectively. Toxicities occurred in $25 \%$ of the GKRS treatments, although the majority were Grade 1 or 2 . At the last follow-up, 4 patients (33\%) had died a neurological death at a median age of 39 years (IQR 37-46 years), and their cases accounted for $45 \%$ of all tumors, $55 \%$ of all treated tumors, and $58 \%$ of all GKRSs. Univariate analysis revealed several predictive variables for distant failure, including male sex ( $\mathrm{HR} 0.28,95 \% \mathrm{Cl} 0.086-0.92, \mathrm{p}=0.036$ ), age at distant failure ( $\mathrm{HR} 0.92,95 \% \mathrm{Cl} 0.90-0.95$, $p<0.0001$ ), and prior number of GKRS treatments (HR 1.2, 95\% Cl 1.1-1.4, $p=0.0049$ ). Local failure, maximum size of the treated tumor, delivered tumor margin dose, and WHO grade were not significant. On multivariate analysis, age at distant failure (HR 0.91, 95\% Cl 0.88-0.95, p < 0.0001) and prior number of GKRSs (HR 1.3, 95\% Cl 1.1-1.5, $p=0.004$ ) remained significant. No malignant transformation events among treated tumors were observed.

CONCLUSIONS Radiosurgery represents a feasible modality with minimal toxicity for NF2-associated meningiomas. Increasing patient age was associated with a decreased rate of distant failure, whereas an increasing number of prior GKRS treatments predicted distant failure. Further studies are necessary to determine the long-term patterns of treatment failure in these patients.

http://thejns.org/doi/abs/10.3171/2014.10.JNS132593

KEY WORDS Gamma Knife; stereotactic radiosurgery; meningioma; neurofibromatosis Type 2; oncology

$\mathrm{N}^{2}$ EUROFIBROMATOSIs Type 2 (NF2) is a rare autosomal dominant disorder predisposing patients to multiple benign nervous system tumors. ${ }^{1}$ While bilateral vestibular schwannomas are considered the hallmark tumors of NF2, meningiomas are the second most frequent tumor type and occur in $45 \%-58 \%$ of patients. ${ }^{1}$ By the age of 70 years, the cumulative incidence of meningioma is close to $80 \% .{ }^{16}$ Progressive meningiomas can lead to focal or generalized seizure disorders and neurological deficits. Moreover, the presence of meningiomas confers a 2.5-fold increase in the relative risk of death in patients with NF2. ${ }^{4}$ While stereotactic radiosurgery (SRS) has been shown to provide satisfactory control rates in sporadic intracranial meningiomas, ${ }^{10}$ to date, no known studies have specifically looked at the use of SRS in patients with NF2.

The current report presents the results of a single-institution case series investigating the use of Gamma Knife radiosurgery (GKRS) for the treatment of NF2-associated meningiomas. We present the clinical outcomes, including the patterns of failure and toxicities, associated with

ABBREVIATIONS GKRS = Gamma Knife radiosurgery; IQR = interquartile range; NF2 = neurofibromatosis Type 2; SRS = stereotactic radiosurgery. SUBMITTED November 27, 2013. ACCEPTED October 30, 2014.

INCLUDE WHEN CITING Published online January 2, 2015; DOI: 10.3171/2014.10.JNS132593.

DISCLOSURE The authors report no conflict of interest concerning the materials or methods used in this study or the findings specified in this paper. 
the treatment of 12 consecutive patients at a single tertiary care cancer center during the period from 1999 to 2013.

\section{Methods \\ Patient Population}

The Wake Forest Baptist Health Institutional Review Board approved this study. The Department of Radiation Oncology Gamma Knife Tumor Registry at Wake Forest Baptist Health was searched for all patients with NF2 who had undergone GKRS for meningioma between January 1, 1999, and September 19, 2013. Patient outcomes were determined using the patients' electronic medical records.

\section{Gamma Knife Procedure}

After patient evaluation by a neurosurgeon and radiation oncologist, informed consent for GKRS is obtained. A 4-pin Leksell stereotactic head frame is placed after applying a local anesthetic. The patient then undergoes highresolution contrast-enhanced stereotactic MRI. Radiosurgery treatment plans are generated using the Leksell GammaPlan system (Elekta AB) and executed using a Leksell model B or C Gamma Knife or Perfexion unit (Elekta AB).

At our institution, the indications for treating meningiomas in patients with NF2 are 1) symptoms from a particular meningioma and/or 2) documented lesion growth. In general, only symptomatic or growing meningiomas are treated during a radiosurgery session. Patients treated with GKRS exhibited evidence of multiple progressive lesions; therefore, resection was a less attractive treatment option since it would require multiple craniotomies.

\section{Patient Follow-Up}

Follow-up clinic visits and MRI examinations are typically conducted 3-6 months after GKRS treatment and then yearly thereafter. Local treatment failure is defined as tumor recurrence within the prescription volume or within $2 \mathrm{~cm}$ of the tumor margin on MRI, as described by Attia et $\mathrm{al}^{2}$ This definition of local failure for meningioma is used because the relevance of fractionated radiation margins of up to $2 \mathrm{~cm}$ has been described for meningioma. Local failure is further differentiated as either central or marginal. Central failure is defined as recurrence within the treatment volume, whereas marginal failure is defined as recurrence within $2 \mathrm{~cm}$ of the treatment volume..$^{14}$ Distant treatment failure is defined as the development of a new tumor more than $2 \mathrm{~cm}$ from the prescription isodose line. In cases with significant disease progression after GKRS treatment, additional stereotactic radiosurgery is often offered. Times to local and distant failure were recorded.

Toxicity grades were assessed using criteria from the Common Terminology Criteria for Adverse Events (version 4.0, http://evs.nci.nih.gov/ftp1/CTCAE/About.html).

Time to death was also recorded if applicable. Death was queried in the Social Security Death Index. Cause of death was determined to be neurological or nonneurological, if possible. Neurological death was defined as stable systemic disease and progressive neurological dysfunction or as simultaneously advancing systemic and neurological dysfunction..$^{13}$

\section{Statistical Analysis}

Descriptive analyses of continuous data are described using the mean and median in the case of normal and nonnormal distributions, respectively, whereas categorical data are described as frequencies. Median follow-up was calculated at the time of data collection for those patients who had not yet died. All time-to-event data were calculated from the time of initial GKRS treatment and are described with Kaplan-Meier plots. Overall survival, local tumor control, and distant tumor control are compared across strata using the log-rank test. Because the majority of meningiomas in this series were WHO Grade I, local and distant tumor control were graphed against and compared with control data for sporadic WHO Grade I meningiomas..$^{12}$ Univariate analysis was first performed to identify potential factors associated with distant failure. Only variables with a $\mathrm{p}$ value $\leq 0.2$ were entered into the multivariate model. A backward stepwise selection method was used to build a multivariate Cox proportional hazards model on a per-tumor basis to determine predictors of distant failure. The multivariate model was built by a priori consideration of the factors for which data were gathered. All computations were performed using SAS version 9.2 (SAS Institute Inc.) and Microsoft Excel 2013.

\section{Results}

Among the 12 patients who met the search criteria, 125 tumors were identified, 87 (70\%) of which were treated with 24 GKRS procedures in the defined study period. The median number of meningiomas treated during the first GKRS session was 3.5 (interquartile range [IQR] 2.85.3). Seven patients (58\%) had only 1 GKRS procedure, whereas 5 patients $(42 \%)$ had multiple procedures. The median prescription dose prescribed to the margin of the enhancing tumor was 12 Gy (IQR 12-14 Gy). Individual patient demographics, tumor characteristics, and GKRS characteristics appear in Table 1. The relevant descriptive statistics are summarized in Table 2.

\section{Treatment}

Gamma Knife radiosurgery treatments were indicated for symptomatic lesions in 4 patients. Symptoms in these patients included 1) increasing gait instability; 2) headaches; 3) seizures, personality changes, increased headaches, nausea, and vomiting; and 4) headaches and gait instability. Two of these patients' symptoms improved with GKRS. The status of the symptoms of the other 2 patients was unknown after GKRS. The remaining 20 treatments were indicated for progressive, asymptomatic meningiomas.

On repeat GKRS, 48 tumors were treated. Of these, 17 (35\%) were new tumors, 26 (54\%) were old tumors, and 5 $(10 \%)$ were unknown.

\section{Survival Data}

At the time of our analysis, 4 patients (33\%) had died and $8(67 \%)$ were alive. As seen in Fig. 1, survival rates at 5 years and 10 years were $100 \%$ and $44 \%$, respectively. Median overall survival was 110 months (IQR 94-125 months). For surviving patients, the overall median time from initial GKRS treatment to the last follow-up was 43 months (IQR 34-110 months). 


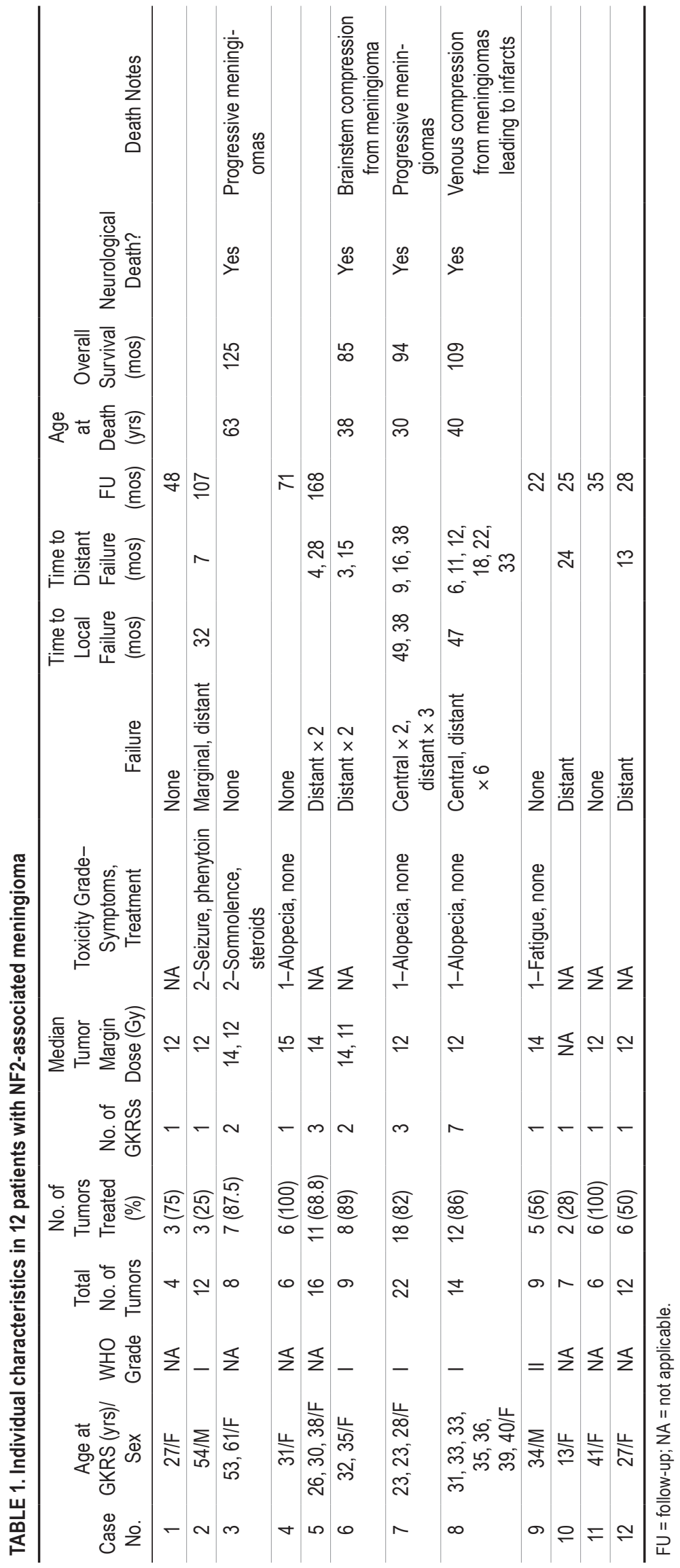


TABLE 2. Summary of patient and treatment characteristics

\begin{tabular}{|c|c|}
\hline Variable & Value \\
\hline Total no. of meningiomas & 125 \\
\hline \multicolumn{2}{|l|}{ No. of meningiomas per patient } \\
\hline Median (IQR) & $9(5.75)$ \\
\hline Range & $4-22$ \\
\hline \% Meningiomas treated (no.) & $70 \%(87)$ \\
\hline Range & $25 \%-100 \%$ \\
\hline \multicolumn{2}{|c|}{ Repeat GKRS: treated meningioma type (no. [\%]) } \\
\hline Total & $48(100)$ \\
\hline Old & $26(54)$ \\
\hline New & $17(35)$ \\
\hline Unknown & $5(10)$ \\
\hline \multicolumn{2}{|l|}{ Location (no. [\%]) } \\
\hline Convexity & $48(38)$ \\
\hline Falcine & $20(16)$ \\
\hline Parasagittal & $19(15)$ \\
\hline Sphenoid & $9(7)$ \\
\hline Posterior fossa & $7(6)$ \\
\hline Tentorium & $6(5)$ \\
\hline Cavernous sinus & $6(5)$ \\
\hline Petroclival & $6(5)$ \\
\hline Olfactory groove & $3(2)$ \\
\hline Cerebellopontine & $1(1)$ \\
\hline \multicolumn{2}{|l|}{ Max long-axis dimension in $\mathrm{cm}$} \\
\hline Median of all tumors & $1.6(1.1)$ \\
\hline Treated median (IQR) & $1.7(1.2)$ \\
\hline Treated range & $0.74-5.38$ \\
\hline Untreated median (IQR) & $1.26(0.7)$ \\
\hline Untreated range & $0.62-2.49$ \\
\hline \multicolumn{2}{|l|}{ No. of GKRSs } \\
\hline Median (IQR) & $1(1.25)$ \\
\hline Range & $1-7$ \\
\hline \% Patients w/ multiple GKRSs (no.) & $42 \%(5)$ \\
\hline \multicolumn{2}{|l|}{ Age at first GKRS in yrs } \\
\hline Median (IQR) & $31(10)$ \\
\hline Range & $13-54$ \\
\hline \multicolumn{2}{|l|}{ Margin dose in Gy } \\
\hline Median (IQR) & $12(2)$ \\
\hline Range & $10-15$ \\
\hline \multicolumn{2}{|l|}{ Time to next GKRS in mos } \\
\hline Median (IQR) & $27(36)$ \\
\hline Average (SD) & $38(32)$ \\
\hline Range & $4-96$ \\
\hline \multicolumn{2}{|l|}{ Distant failure } \\
\hline \% GKRSs (no.) & $67 \%(16)$ \\
\hline \% Patients (no.) & $50 \%(6)$ \\
\hline \multicolumn{2}{|l|}{ Time to distant failure in mos } \\
\hline Median (IQR) & $14(14)$ \\
\hline Range & $4-29$ \\
\hline
\end{tabular}

TABLE 2. Summary of patient and treatment characteristics (continued)

\begin{tabular}{|c|c|}
\hline Variable & Value \\
\hline \multicolumn{2}{|l|}{ Marginal failure } \\
\hline \% GKRSs (no.) & $4.2 \%(1$ of 24$)$ \\
\hline \% Treated tumors (no.) & $1.1 \%(1$ of 87$)$ \\
\hline$\%$ Patients (no.) & $8.3 \%$ (1 of 12$)$ \\
\hline Time to marginal failure in mos (range) & $33(33-33)$ \\
\hline \multicolumn{2}{|l|}{ Central failure } \\
\hline \% GKRSs (no.) & $8.3 \%(2$ of 24$)$ \\
\hline \% Treated tumors (no.) & $3.4 \%$ (3 of 87$)$ \\
\hline$\%$ Patients (no.) & $16.7 \%(2$ of 12$)$ \\
\hline Time to central failure in mos (range) & $47.8(39-50)$ \\
\hline \multicolumn{2}{|l|}{ Time to toxicity in mos } \\
\hline Median (IQR) & $4.3(7.8)$ \\
\hline Range & $0.7-30$ \\
\hline \multicolumn{2}{|c|}{ Overall median time from GKRS to last follow-up } \\
\hline Median (IQR) & $43(76)$ \\
\hline Range & $22-171$ \\
\hline$\%$ Died (no.) & $33.3 \%(4)$ \\
\hline \multicolumn{2}{|l|}{ Age at death in yrs } \\
\hline Median (IQR) & $39(9)$ \\
\hline Range & $31-64$ \\
\hline$\%$ Deaths due to neurological death & 100 \\
\hline \multicolumn{2}{|l|}{ Time to death since first GKRS in mos } \\
\hline Median (IQR) & $103(22)$ \\
\hline Range & $87-127$ \\
\hline
\end{tabular}

All patients who died had a neurological death due to progressive meningiomas. Median age at the time of death was 39 years (IQR 37-46 years). The median time to neurological death from initial GKRS treatment was 103 months (IQR 93-115 months). The cases of the 4 patients who died accounted for $45 \%$ of all tumors, $55 \%$ of all treated tumors, and $58 \%$ of all GKRS treatments. One patient died because of brainstem compression from a previously treated meningioma. A second patient died because of compression from a previously treated meningioma causing venous congestion, which subsequently led to an infarct. Specific details about the cause of death were unknown in the other 2 patients, although progressive meningiomatosis led to a worsened performance status and ultimately hospice placement for both.

\section{Patterns of Failure}

Local treatment failure occurred in $4(5 \%)$ of 87 treated tumors from 3 GKRS treatments in 3 patients after GKRS: 3 tumors centrally within the prescription volume and 1 tumor marginally within $2 \mathrm{~cm}$ of the prescription volume. Patients in these cases had a median time to local treatment failure of 43 months (IQR 38-48 months). The tumor with marginal failure was treated with resection. Two of the 3 tumors with central failure were treated using resection, and the third tumor was treated with repeat GKRS. Local tumor control was defined as the absence of either central or marginal tumor progression. The local 


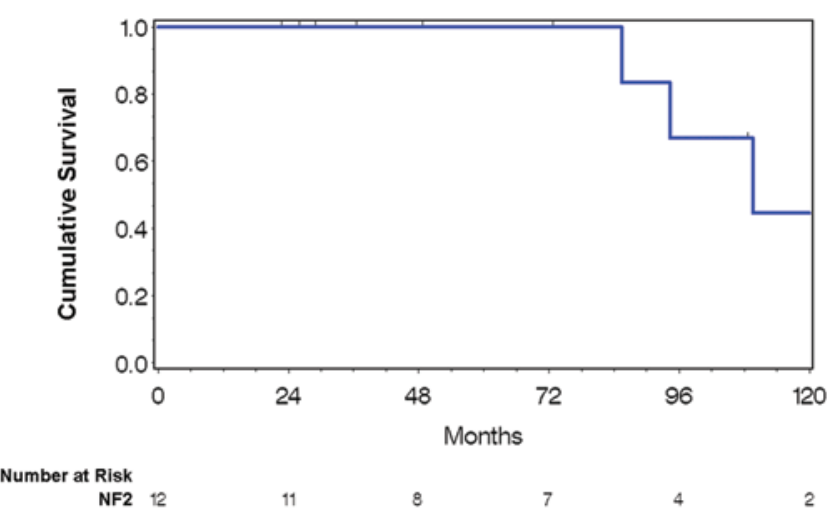

FIG. 1. Overall survival since the first GKRS. Figure is available in color online only.

tumor control rates were $100 \%, 98 \%$, and $92 \%$ at 1,3 , and 5 years, respectively.

Figure 2 shows the Kaplan-Meier curve for local control of NF2-associated meningiomas compared with that for sporadic WHO Grade I meningiomas. Local control at 5 years was $92 \%$ and $88 \%$, respectively. The difference was significant using the log-rank test $(\mathrm{p}<0.001)$.

Sixteen $(67 \%)$ of 24 treatments resulted in distant failure. Patients in these cases had a median time of 14 months (IQR 9-23 months) to distant treatment failure. The distant failure rates were $26 \%, 53 \%, 77 \%$, and $77 \%$ at 1,2 , 5 , and 10 years, respectively. Eight of 16 distant failures were eventually treated with repeat GKRS, and the other 8 instances of distant treatment failure were monitored only.

Figure 3 shows the Kaplan-Meier curve for distant failure for NF2-associated meningiomas compared with that for sporadic Grade I meningiomas. Distant control at 5 years was $23 \%$ for NF2 and $90 \%$ for sporadic Grade I. The difference was significant using the log-rank test $(\mathrm{p}<0.001)$.

\section{Predictors of Distant Failure}

Univariate analysis revealed several predictive variables for distant failure, including male sex (HR 0.28, 95\% CI 0.086-0.92, $\mathrm{p}=0.036)$, age at distant failure $(\mathrm{HR}$

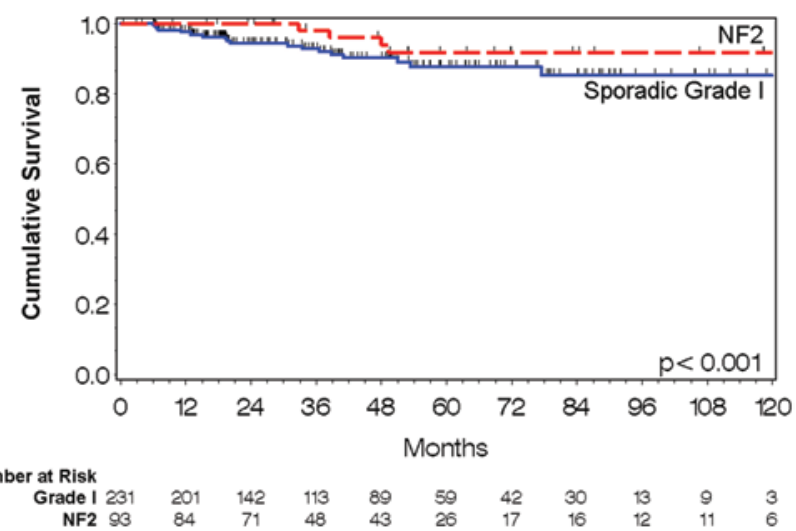

FIG. 2. Kaplan-Meier curve for local control of NF2-associated tumors as compared with sporadic WHO Grade I meningiomas. Local control at 5 years was $92 \%$ for NF2 and $88 \%$ for sporadic Grade I. The differences were significant using the log-rank test $(p<0.001)$. Ninety-three tumors were analyzed given that 6 of the 87 were treated twice with GKRS. Figure is available in color online only.

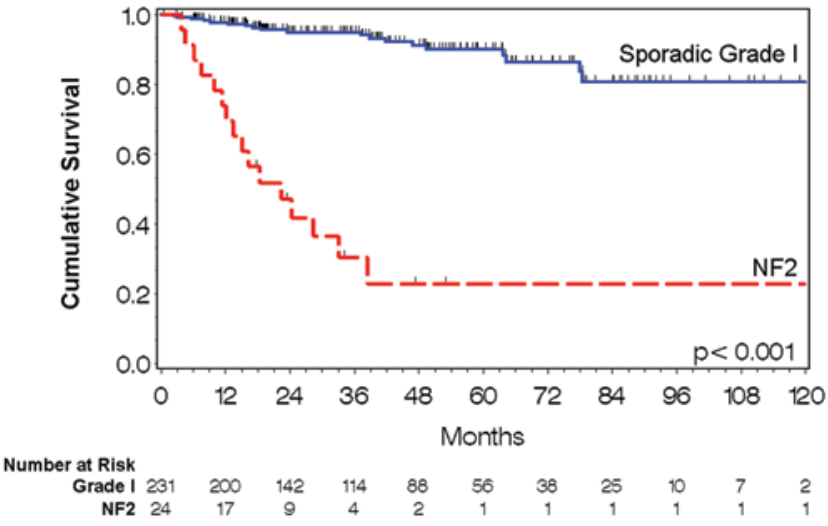

FIG. 3. Kaplan-Meier curve for distal control of NF2-associated tumors compared with sporadic WHO Grade I meningiomas. Distant control at 5 years was $23 \%$ for NF2 and $90 \%$ for sporadic Grade I. The differences were significant using the log-rank test $(p<0.001)$. Figure is available in color online only.

$0.92,95 \%$ CI $0.90-0.95, \mathrm{p}<0.0001)$, and prior number of GKRS treatments (HR 1.2, 95\% CI 1.1-1.4, $\mathrm{p}=0.0049$; Table 3). Local failure, maximum dimension of the treated tumor, tumor margin dose delivered, and WHO grade were not significant.

On multivariate analysis, age at distant failure (HR $0.91,95 \%$ CI $0.88-0.95, \mathrm{p}<0.0001$ ) and prior number of GKRSs (HR 1.3, 95\% CI 1.1-1.5, p = 0.004; Table 4) remained significant.

\section{Malignant Transformation}

There was no malignant transformation of benign tumors in any of the patients following GKRS treatment.

\section{Toxicity}

Six $(25 \%)$ of the 24 treatments resulted in adverse toxicity events. The median time to onset of toxicity was 4.3 months (IQR 1-9 months). Three patients experienced untreated alopecia (Grade 1); 1 patient, untreated fatigue (Grade 1); 1 patient, somnolence due to increased cerebral edema treated with steroids (Grade 2); and 1 patient, seizures treated with phenytoin (Grade 2). Only 1 patient had toxicity-associated adverse changes on MRI with and without contrast. The patient experiencing somnolence had increased edema of the right temporal lobe with mass effect. None of the patients had contrast changes consistent with radiation necrosis.

\section{Adjunctive Treatment}

Two patients underwent resection of tumors initially treated with GKRS. Two patients underwent repeat GKRS

TABLE 3. Hazard ratios of distant failure for patient and treatment characteristics

\begin{tabular}{llcc}
\hline \multicolumn{1}{c}{ Variable } & $\mathrm{HR}$ & $95 \% \mathrm{Cl}$ & $\mathrm{p}$ Value \\
\hline Male & 0.28 & $0.086-0.92$ & 0.036 \\
\hline Age at distant failure $(\mathrm{yrs})$ & 0.92 & $0.90-0.95$ & $<0.0001$ \\
\hline No. of prior GKRSs & 1.2 & $1.1-1.4$ & 0.0049 \\
\hline Max size of treated tumor $(\mathrm{cm})$ & 1.3 & $1.0-1.7$ & 0.0515 \\
\hline Margin dose (Gy) & 0.76 & $0.57-1.0$ & 0.0666 \\
\hline
\end{tabular}


TABLE 4. Cox proportional hazards model for distant failure

\begin{tabular}{llcc}
\hline \multicolumn{1}{c}{ Variable } & $\mathrm{HR}$ & $95 \% \mathrm{Cl}$ & $\mathrm{p}$ Value \\
\hline Age at distant failure $(\mathrm{yrs})$ & 0.91 & $0.88-0.95$ & $<0.0001$ \\
\hline No. of prior GKRSs & 1.3 & $1.1-1.5$ & 0.0035 \\
\hline Max size of treated tumor $(\mathrm{cm})$ & 1.1 & $0.78-1.6$ & 0.60 \\
\hline
\end{tabular}

for enlarging or symptomatic meningiomas. One patient received a ventriculoperitoneal shunt after initial GKRS for hydrocephalus that was present before radiosurgical treatment. None of our patients received bevacizumab as adjunctive therapy.

\section{Discussion}

Treatment options for NF2-associated intracranial meningiomas include conservative observation, surgery, radiation, and chemotherapy. The optimal type and timing of treatment has not yet been determined, as the natural history of the tumors has only recently been described. Dirks et al. were the first to examine long-term growth patterns, new tumor development, and clinical characteristics of NF2-associated meningiomas..$^{5}$ The lesions in their study displayed substantial variability in growth rate and pattern, even among tumors within the same patient. ${ }^{5}$ The majority of meningiomas exhibited saltatory growth patterns with a strong correlation between periods of quiescence and a longer duration of follow-up. ${ }^{5}$ These periods of quiescence have significant implications for clinical management, timing of interventions, and evaluation of the efficacy of therapeutic interventions. ${ }^{5}$

One of the controversies surrounding the radiotherapeutic management of patients with NF2 is the concern about the possibility of malignant transformation of the treated tumor. In a comparison of patient cohorts that did not and did receive radiotherapy (including radiosurgery), it was estimated that $5 \%$ of the patients who had undergone radiosurgery developed radiation-associated malignant transformation. ${ }^{3}$ It is notable, however, that most of the cases of malignant transformation postradiosurgery consisted of the development of malignant peripheral nerve sheath tumor after the treatment of vestibular schwannoma. In the current series, there were no cases of malignant transformation.

Median age at the time of the first GKRS treatment in our study was 31 years, which is much younger than the median age of 57 years in those undergoing sporadic meningioma radiosurgery. ${ }^{15}$ The indications for treatment at our institution included symptoms caused by a lesion or imaging-documented progression in lesion size. Most of the meningiomas were symptomatic or had progressed, requiring GKRS treatment; ${ }^{13}$ however, 38 tumors remained asymptomatic or stable in size and thus did not require treatment. These tumors may be temporarily quiescent and eventually require resection or radiosurgery. Additional radiosurgery was indicated due to newly symptomatic or progressive tumors in 5 patients (42\%), compared with the $3 \%-4 \%$ rate seen in sporadic meningiomas (both benign and malignant). ${ }^{10,15}$ Two patients had previously treated but progressive tumors $(n=6)$ that were retreated with GKRS. Not surprisingly, the average time to the next treatment in patients with NF2 was shorter at 38 months, compared with an average of 49 months in patients with sporadic meningiomas. ${ }^{10} \mathrm{~A}$ single patient $(8.3 \%)$ required resection of 3 WHO Grade I tumors following GKRS treatment, 2 of which had failed centrally.

A number of studies have looked at radiosurgery for benign sporadic meningioma and reported high tumor control rates ranging from $93 \%$ to $96 \%$ at 5 years..$^{7,9,17}$ However, atypical and malignant histology have been reported in $29 \%$ and $6 \%$ of surgical NF2-associated meningioma specimens, respectively. ${ }^{8}$ Higher-grade meningiomas are known to have a higher rate of local treatment failure after radiosurgery. ${ }^{2}$ We found the local tumor control rate to be similar to that for benign meningiomas, $92 \%$ at 5 years, although our sample size was small. More specifically, we found that local control of NF2-associated meningiomas was similar to that of sporadic Grade I meningiomas.

Seven $(58 \%)$ of 12 patients demonstrated new tumors that had not been present on initial MRI, which is similar to the rate of $65 \%$ cited in previous studies looking at the natural history of NF2-associated meningiomas. ${ }^{5}$ The distant control rate was significantly lower than that for sporadic Grade I meningiomas. Male sex, which is a risk factor for treatment failure in sporadic meningiomas, ${ }^{12}$ was associated with a lower rate of distant failure in NF2. Increasing age was associated with a lower rate of distant failure as well, and this may be attributable to the fact that patients are censored by their own death and have less time during which they are at risk for distant failure. Additionally, this finding is consistent with the fact that a younger age at presentation with NF2 is associated with poorer outcomes. Given the disease characteristics and natural history of NF2, it is not surprising that an increasing number of prior GKRSs was predictive of distant treatment failure. Given the higher rate of high-grade meningiomas in the NF2 population, it may be prudent to consider higher dosing to the tumor to treat for a presumably higher-grade tumor. This practice has been used successfully in other clinical scenarios, such as radiation-induced tumors, in which the likelihood that a meningioma is a higher-grade tumor is greater. ${ }^{11}$

While 6 patients $(50 \%)$ suffered toxicity following GKRS, the majority of symptoms were mild, categorized as Grade 1, and did not have accompanying adverse changes on MRI. Additionally, toxicities occurred in $25 \%$ of all GKRS treatments.

Four $(33 \%)$ of our patients died at a median age of 39 years. These 4 patients accounted for $45 \%$ of all tumors, $55 \%$ of the treated tumors, and $58 \%$ of all GKRS treatments. Of note, all patients with central failure eventually died, with a median time of 36 months from failure to death. All causes of death were categorized as neurological death, typically due to tumor compression of the brain. Interestingly, in 1 patient, the compression caused venous congestion, leading to cerebral infarcts and causing death. Our results are consistent with what has been reported in the scientific literature, as patients with NF2 (diagnosed prior to the era of genetic testing) generally have a life expectancy of 15 years from the time of diagnosis. ${ }^{6}$

As expected, the nature of NF2 predisposes patients to treatment at a younger age, a greater need for additional treatment, and a shorter time to the next treatment. Given the large tumor burden that these patients can develop and 
the saltatory growth patterns of the meningiomas, resection of growing tumors in the absence of symptoms may not be indicated ${ }^{13}$ Radiosurgery may be an alternative in these cases, as it has high tumor control rates and limited toxicity. Its use, however, may need to be weighed against the theoretical risk of malignant transformation, such that radiosurgery may be best used in cases of persistent or significant growth or symptomatic tumors.

There are several limitations to this study. Because NF2-associated tumors have been shown to have periods of quiescence, ${ }^{5}$ a major limitation of this study is the relatively short follow-up period. Although our median follow-up of 43 months is longer than the average quiescent period of 29 months, as described by Dirks et al., ${ }^{5}$ the follow-up period may still be too short to observe any malignant progression. Another limitation is the small number of patients and tumors, making generalization difficult. The 4 instances of marginal and central failure were insufficient for statistical analysis. Because all patients were treated at a single institution, our findings may not be generalizable to other populations. As a tertiary referral center, it is also possible that patients with more aggressive disease were referred to us, resulting in a selection bias.

Finally, the study may be limited by potential changes in the treatment algorithm over the interval of the study. In the past decade, the treatment dose for meningioma has decreased from 16 Gy to about 12 Gy. ${ }^{12}$ Another change is the emergence of bevacizumab for acoustic schwannoma in NF2, although none of our patients were placed on bevacizumab. Lastly, we may be more willing now to treat patients with NF2, compared with a decade ago. A long-term prospective trial would be the optimal means of determining tumor control rates and malignant transformation rates after treatment with radiosurgery.

\section{Conclusions}

Radiosurgery represents a feasible modality for NF2associated meningiomas. Increasing age was associated with a decreased rate of distant failure, whereas a greater number of GKRS treatments was predictive of distant failure. Further studies are necessary to determine the longterm patterns of failure in these patients.

\section{Acknowledgment}

This publication would not have been possible without the mentorship and guidance of Thomas L. Ellis, MD. We cherish the opportunities we had to work with him and remember his passion for the education of medical students and residents in neurosurgery. We hope to continue his legacy of courage, determination, grace, and unselfishness.

\section{References}

1. Asthagiri AR, Parry DM, Butman JA, Kim HJ, Tsilou ET, Zhuang Z, et al: Neurofibromatosis type 2. Lancet 373:19741986, 2009

2. Attia A, Chan MD, Mott RT, Russell GB, Seif D, Daniel Bourland J, et al: Patterns of failure after treatment of atypical meningioma with gamma knife radiosurgery. J Neurooncol 108:179-185, 2012

3. Baser ME, Evans DG, Jackler RK, Sujansky E, Rubenstein A: Neurofibromatosis 2, radiosurgery and malignant nervous system tumours. Br J Cancer 82:998, 2000

4. Baser ME, Friedman JM, Aeschliman D, Joe H, Wallace AJ,
Ramsden RT, et al: Predictors of the risk of mortality in neurofibromatosis 2. Am J Hum Genet 71:715-723, 2002

5. Dirks MS, Butman JA, Kim HJ, Wu T, Morgan K, Tran AP, et al: Long-term natural history of neurofibromatosis Type 2-associated intracranial tumors. J Neurosurg 117:109-117, 2012

6. Evans DG, Huson SM, Donnai D, Neary W, Blair V, Newton V, et al: A clinical study of type 2 neurofibromatosis. Q J Med 84:603-618, 1992

7. Friedman WA, Murad GJ, Bradshaw P, Amdur RJ, Mendenhall WM, Foote KD, et al: Linear accelerator surgery for meningiomas. J Neurosurg 103:206-209, 2005

8. Goutagny S, Bah AB, Henin D, Parfait B, Grayeli AB, Sterkers O, et al: Long-term follow-up of 287 meningiomas in neurofibromatosis type 2 patients: clinical, radiological, and molecular features. Neuro Oncol 14:1090-1096, 2012

9. Kondziolka D, Lunsford LD, Coffey RJ, Flickinger JC: Gamma knife radiosurgery of meningiomas. Stereotact Funct Neurosurg 57:11-21, 1991

10. Kondziolka D, Mathieu D, Lunsford LD, Martin JJ, Madhok $\mathrm{R}$, Niranjan A, et al: Radiosurgery as definitive management of intracranial meningiomas. Neurosurgery 62:53-60, 2008

11. Kuhn EN, Chan MD, Tatter SB, Ellis TL: Gamma knife stereotactic radiosurgery for radiation-induced meningiomas. Stereotact Funct Neurosurg 90:365-369, 2012

12. Kuhn EN, Taksler GB, Dayton O, Loganathan AG, Vern-Gross TZ, Bourland JD, et al: Patterns of recurrence after stereotactic radiosurgery for treatment of meningiomas. Neurosurg Focus 35(6):E14, 2013

13. Patchell RA, Tibbs PA, Regine WF, Dempsey RJ, Mohiuddin $\mathrm{M}$, Kryscio RJ, et al: Postoperative radiotherapy in the treatment of single metastases to the brain: a randomized trial. JAMA 280:1485-1489, 1998

14. Paulsson AK, McMullen KP, Peiffer AM, Hinson WH, Kearns WT, Johnson AJ, et al: Limited margins using modern radiotherapy techniques does not increase marginal failure rate of glioblastoma. Am J Clin Oncol 37:177-181, 2014

15. Pollock BE, Stafford SL, Link MJ, Brown PD, Garces YI, Foote RL: Single-fraction radiosurgery of benign intracranial meningiomas. Neurosurgery 71:604-613, 2012

16. Smith MJ, Higgs JE, Bowers NL, Halliday D, Paterson J, Gillespie J, et al: Cranial meningiomas in 411 neurofibromatosis type 2 (NF2) patients with proven gene mutations: clear positional effect of mutations, but absence of female severity effect on age at onset. J Med Genet 48:261-265, 2011

17. Stafford SL, Pollock BE, Foote RL, Link MJ, Gorman DA, Schomberg PJ, et al: Meningioma radiosurgery: tumor control, outcomes, and complications among 190 consecutive patients. Neurosurgery 49:1029-1038, 2001

\section{Author Contributions}

Conception and design: Liu, Kuhn, Lucas, Tatter, Chan. Acquisition of data: Liu, Kuhn. Analysis and interpretation of data: Liu, Lucas, Chan. Drafting the article: Liu, Lucas, Chan. Critically revising the article: all authors. Reviewed submitted version of manuscript: all authors. Approved the final version of the manuscript on behalf of all authors: Liu. Statistical analysis: Liu, Lucas, Chan. Study supervision: Tatter, Chan.

\section{Supplemental Information \\ Current Affiliation}

Dr. Kuhn: Department of Neurosurgery, University of Alabama at Birmingham Medical Center, Birmingham, AL.

\section{Correspondence}

Ann Liu, Department of Neurosurgery, Wake Forest School of Medicine, Medical Center Blvd., Winston-Salem, NC 27157. email: aliu@wakehealth.edu. 